\title{
Preferência alimentar e desempenho de Brontocoris tabidus Signoret (Hemiptera, Pentatomidae) em plantas hospedeiras
}

\author{
Roberta R. Coelho ${ }^{1}$, Antônio F. S. L. Veiga ${ }^{2}$ \& Jorge B. Torres ${ }^{1}$
}

${ }^{1}$ Programa de Pós-Graduação em Entomologia Agrícola, Universidade Federal Rural de Pernambuco, Av. Dom Manoel de Medeiros s/n, Dois Irmãos, 52171-900 Recife-PE, Brasil. coelho.rr@gmail.com.

${ }^{2}$ Departamento de Biologia-Zoologia, Universidade Federal Rural de Pernambuco, Av. Dom Manoel de Medeiros s/n, Dois Irmãos, 52171-900 Recife-PE, Brasil. afsouzaleao@click21.com.br.

\begin{abstract}
Feeding preference and performance of Brontocoris tabidus (Signoret) (Hemiptera, Pentatomidae) among host plants. The choice between cotton, Gossypium hirsutum L. (Malvaceae), green bean, Phaseolus vulgaris L. (Fabaceae) and pigweed, Amaranthus lividus L. (Amaranthaceae) and its relationship as first feeding, weight gain and biological performance of the predator Brontocoris tabidus (Signoret) (Hemiptera, Pentatomidae) adults were studied. Adults of the predator were allowed to choose between plants and, immediately paired and reared on the same selected plants plus prey. Furthermore, nymphal development and adult stage were obtained on the same plants plus prey. The results show that B. tabidus do not exhibit host plant preference among tested plants. Likewise, feeding period (106 to 115 minutes) and weight gain (28 to $30 \%$ of body weight) were statistically equal among plants. However, predators at adult stage only or nymphal and adult stages maintained on young green bean plants plus prey exhibited lower performance compared to the other plants. Nymphs feeding exclusively on plants lived, on average, up to 6.5 days but did not reach adult stage and lived longer on cotton plants and pig weed. The results indicated that adults of the predator $B$. tabidus do not exhibit selection on host plant to feed although different results in its life history was attained among the studied plants with better results for cotton and pigweed.
\end{abstract}

KEYWORDS. Feeding behavior; phytophagy; predatory stinkbug; reproductive output.

RESUMO. Preferência para alimentação e desempenho de Brontocoris tabidus Signoret (Hemiptera, Pentatomidae) em plantas hospedeiras. O comportamento de escolha entre plantas pelo percevejo predador Brontocoris tabidus (Signoret) (Hemiptera, Pentatomidae) e a relação com o ganho de peso e seu desempenho foram investigados com plantas de algodoeiro, Gossypium hirsutum L. (Malvaceae), caruru, Amaranthus lividus L. (Amaranthaceae) e feijão, Phaseolus vulgaris L. (Fabaceae). Adultos do predador foram permitidos livre escolha entre plantas e, posteriormente, mantidos sobre as mesmas plantas escolhidas inicialmente para a alimentação. Também, o desenvolvimento e a reprodução foram obtidos confinando os predadores com e sem presa mais plantas. Os resultados mostraram que B. tabidus não demonstrou preferência para alimentação entre as plantas estudadas. O tempo de alimentação (106 a 115 minutos) e ganho de peso (28 a 30\% do peso vivo) foram similares em relação à alimentação em plantas. Entretanto, a longevidade das fêmeas foi menor quando mantidas em feijão, comparadas com algodoeiro e caruru. A duração da fase ninfal do predador variou entre as plantas, bem como a produção de descendentes demonstrando menor desempenho sobre plantas de feijão. As ninfas alimentadas, apenas, de plantas não atingiram a fase adulta e viveram até 6,5 dias, sendo maior a sobrevivência em algodoeiro e caruru. Os resultados mostram que apesar de B. tabidus não apresentar seleção da planta hospedeira para alimentação, estas podem contribuir diferentemente na sua história de vida com destaque para o algodão e o caruru entre as plantas estudadas.

PALAVRAS-CHAVE. Comportamento de escolha; desempenho reprodutivo; fitofagia; percevejo predador.

A dinâmica populacional de pragas e de seus inimigos naturais usualmente se dá através de uma assincronia que resulta em surtos populacionais das pragas e, somente, tardiamente ocorrem predadores em número suficiente para a regulação populacional. Isto se deve, dentre outros fatores, à ausência de predadores nas proximidades para a colonização das lavouras e, posteriormente, à falta de alimento para a sustentação dos inimigos naturais colonizando as lavouras. Diante disso, o conhecimento da estrutura de teias alimentares em ecossistemas agrícolas é fundamental para a escolha do inimigo natural e deve incluir a possibilidade de seu estabelecimento e manutenção em campo, mesmo em períodos de escassez de presas.

A complementação da alimentação com seiva das plantas ou de pólen e néctar pode facilitar a permanência de predadores zoofitófagos nos agroecossistemas (Jervis \& Kidd 1996). A maioria dos percevejos predadores apresenta zoofitofagia em algum estágio de sua vida (Cohen 1996) e, ao se alimentar de plantas, podem se manter nas lavouras durante períodos de escassez de presas (Naranjo \& Gibson 1996). Sendo assim, a alta disponibilidade de material vegetal no habitat pode permitir a alimentação alternada entre planta e presa pelos zoofitófagos, dependendo da densidade da presa (Coll \& Guershon 2002).

Vários percevejos predadores neotropicais têm sido alvos de estudos sobre fitofagia, como é o caso de Orius insidiosus (Say, 1832) (Armer et al. 1998), Geocoris punctipes (Say, 1831) (Tillman \& Mullinix Júnior 2003), Podisus nigrispinus (Dallas, 1851) (Evangelista Júnior et al. 2004), Supputius cinctipes (Stal, 1860) (Assis-Júnior et al. 1998) e Brontocoris tabidus (Signoret, 1863) (Zanuncio et al. 2000). Estes predadores têm 
sido favorecidos quando têm material vegetal associado à dieta de presas. Entre os benefícios, pode-se citar a redução do período ninfal, maior peso de adultos, ganho em fecundidade e longevidade (Stoner et al. 1974; Naranjo \& Stimac 1985; Ruberson et al. 1986; Zanuncio et al. 2000; Evangelista-Júnior et al. 2004).

A digestão extra-oral de percevejos predadores torna necessária a escolha do alimento (Legaspi \& Legaspi Jr 2004) por exigir a produção de toxinas para neutralizar a presa e enzimas para digerir o alimento antes de sua ingestão. Esse comportamento de alimentação melhora a história de vida de B. tabidus com presa e planta (Barcelos et al. 1994; Zanuncio et al. 2006), o que torna esse predador um organismo importante para estudos de zoofitofagia. Além disso, apresentam fácil manipulação por ter tamanho relativamente maior $(230 \mathrm{mg})$ (Oliveira et al. 2005) que outros percevejos predadores e maior produção de ovos em condições adequadas de alimento (Zanuncio et al. 2006). Dessa forma, B. tabidus exige grande quantidade de alimento (Oliveira et al. 2005) o que, consequentemente, pode resultar em maior dependência da planta para complementar sua alimentação e obter água via seiva.

$\mathrm{O}$ algodoeiro e o feijoeiro hospedam diversas lagartas desfolhadoras alvo de percevejos predadores, enquanto que o caruru é uma planta daninha comum nesses agroecossistemas e que, também, hospeda diversos lepidópteros que podem ser presas alternativas para os predadores ou mesmo como planta hospedeira alternativa para esses predadores se alimentarem. Estudos prévios com o percevejo predador $P$. nigrispinus, demonstraram que plantas de algodoeiro, caruru e feijão foram favoráveis ao seu desenvolvimento, mantendo alta sobrevivência ninfal e fecundidade das fêmeas, quando comparados aos insetos sem acesso a plantas em sua dieta (Molina-Rugama et al. 1997; Oliveira et al. 2002; Evangelista-Júnior et al. 2004). Assim, este trabalho estudou o comportamento de escolha de $B$. tabidus entre plantas para alimentação e, se isto, está associado ao seu desempenho biológico. A hipótese de que B. tabidus prefere se alimentar na planta onde obterá melhor desempenho foi investigado com plantas de algodoeiro [Gossypium hirsutum L. (Malvaceae)], feijão [Phaseolus vulgaris L. (Fabaceae)] e de caruru [Amaranthus lividus L. (Amaranthaceae)].

\section{MATERIALE MÉTODOS}

Criação dos insetos. Adultos do percevejo predador, $B$. tabidus, utilizados nos experimentos foram oriundos da colônia mantida no Laboratório de Controle Biológico e Ecologia de Insetos da Universidade Federal Rural de Pernambuco (UFRPE). A criação foi mantida em potes plásticos de 1L com tampa possuindo uma abertura circular fechada com tela de náilon de $2 \mathrm{~mm}$ de malha para facilitar a ventilação. Tanto os adultos como as ninfas foram alimentados com pupas de Tenebrio molitor L., 1785 (Coleoptera, Tenebrionidae) e vagem de feijão, Phaseolus vulgaris. A criação de T. molitor foi mantida em bandejas contendo farelo de trigo, levedura de cerveja e com pedaços de legumes oferecidos semanalmente como fonte de umidade e nutrientes. Maiores detalhes da criação de percevejos predadores e a da presa alternativa, $T$. molitor, podem ser obtidas em Torres et al. (2006). A criação dos predadores, presas e condução dos experimentos foram realizados em laboratório com controle de temperatura (média de $25 \pm 2{ }^{\circ} \mathrm{C}$ ), fotofase de $12 \mathrm{~h}$ e umidade relativa de $72 \pm 12 \%$.

Plantas utilizadas. Foram utilizadas plantas de algodoeiro, G. hirsutum, cultivar Acala 90, feijão, P. vulgaris, cultivar Macarrão (Horticeres ${ }^{\circledR}$ Sementes, Campinas, SP) e de caruru, A. lividus. As sementes de caruru foram coletadas na horta do Departamento de Agronomia da UFRPE, enquanto as sementes de algodoeiro foram cedidas pela Monsanto, São Paulo, SP, e de feijão adquirida em casa agropecuária em Recife, PE. As plantas foram cultivadas em potes plásticos de $250 \mathrm{~mL}$ contendo mistura de solo e húmus na proporção de 4:1 mais adubo formulado NPK 4-14-8 com aproximadamente 8 a 10g por $1 \mathrm{Kg}$ de mistura de substrato. Adubações com uréia diluída em água, na concentração de $5 \mathrm{~g} . \mathrm{L}^{-1}$, foram feitas semanalmente. O plantio foi realizado semanalmente para a obtenção de plantas de, aproximadamente, 20 dias de idade (em torno de $18 \mathrm{~cm}$ de altura) durante todo o experimento.

Comportamento de escolha da planta por $B$. tabidus e efeito na reprodução. Foi estudado se o percevejo predador $B$. tabidus apresenta comportamento de escolha por plantas hospedeiras para alimentação. Assim, plantas de algodoeiro, feijão e caruru foram expostas a insetos adultos em gaiolas retangulares de acrílico transparentes com dimensões de $40 \times 50 \times 50 \mathrm{~cm}$. Foi utilizado um total de 100 machos e 94 fêmeas para o estudo de escolha entre as plantas hospedeiras. Os predadores foram identificados com etiquetas numeradas, fixadas no pronoto e mantidos sem alimento e água por $24 \mathrm{~h}$ para homogeneizar a condição de saciação. Antes da oferta das plantas aos insetos, os predadores foram pesados e liberados na densidade de cinco predadores por gaiola contendo as três plantas. Após a liberação, os predadores foram monitorados quanto à escolha da planta (i), tempo de alimentação (ii) e ganho de peso (iii), esta última característica determinada pela diferença de peso antes e após alimentação na planta. A frequiência de escolha por planta hospedeira foi determinada a partir dessa escolha.

A avaliação foi contínua por um período de $12 \mathrm{~h}$ (07:00 as 19:00h) para a verificação da preferência do inseto pela planta para alimentação, tempo de alimentação e ganho de peso. Ao término do período de oferta da planta para alimentação, foram formados casais com indivíduos que escolheram a mesma planta e mantidos sob a planta escolhida durante a fase adulta até a morte. O confinamento na fase adulta foi feito em gaiolas plásticas transparentes de $2 \mathrm{~L}$ de volume tipo "PET" com orifícios circulares na parte mediana e fechados com tela de metal com $2 \mathrm{~mm}$ de malha permitindo a ventilação no interior das gaiolas. Cada gaiola foi vertida sobre as plantas cultivadas em potes plásticos de $250 \mathrm{ml}$. Pupas de T. molitor foram oferecidas aos predadores em recipientes confeccionados com tela plástica pendurados no interior das gaiolas. Os insetos e 

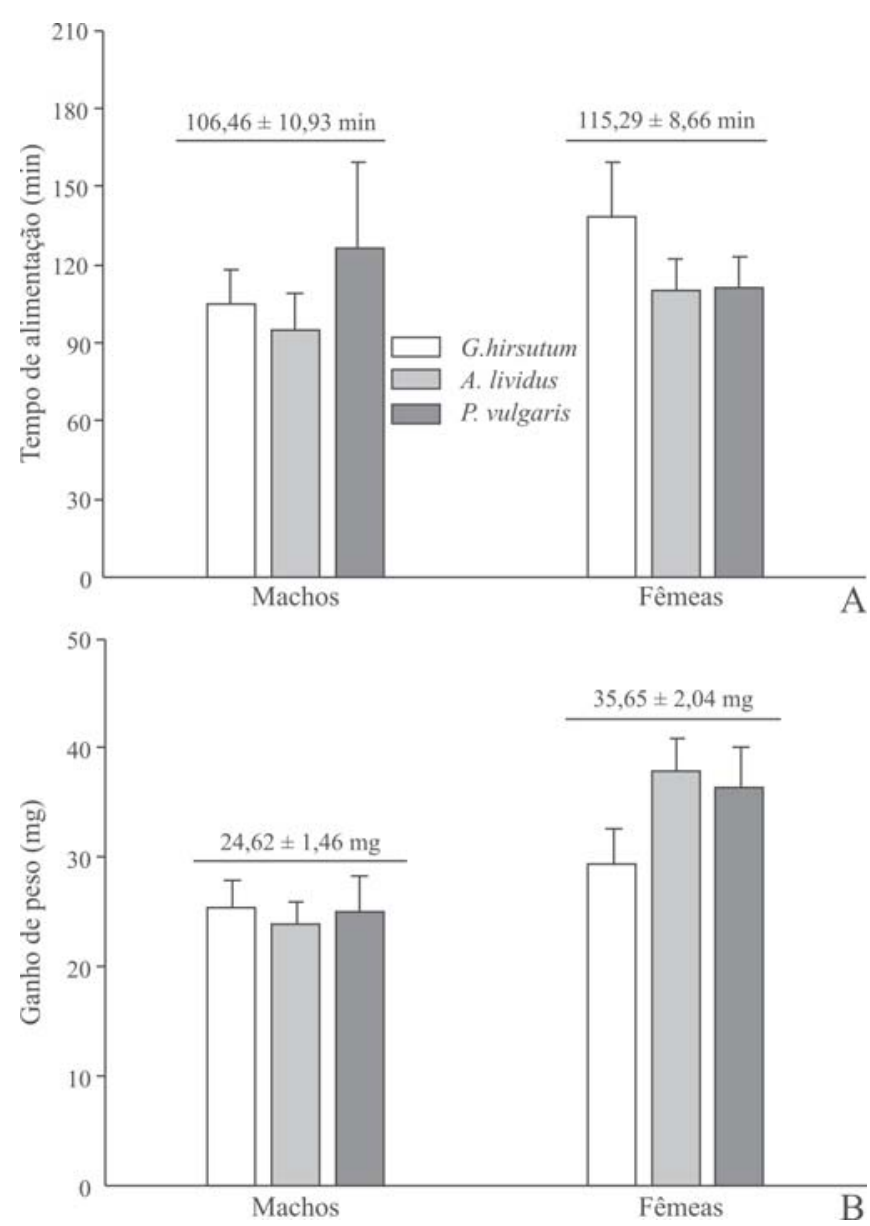

Fig. 1. Tempo de alimentação (minutos) (A) e ganho de peso (miligrama) (B) de machos e fêmeas de Brontocoris tabidus em Gossypium hirsutum, Amaranthus lividus e Phaseolus vulgaris após 24h sem alimento.

plantas foram mantidos em câmara climáticas do tipo B.O.D. à temperatura de $25 \pm 1{ }^{\circ} \mathrm{C}, 68 \pm 5 \%$ de U.R. e fotofase de $12 \mathrm{~h}$. Vinte e quatro casais foram formados, por espécie de planta, com 12 recebendo pupas de $T$. molitor em abundância e 12 mantidos sem essa presa e com acesso, apenas, à planta escolhida para alimentação.

As avaliações, durante a fase adulta, foram feitas a cada 48h quando as posturas eram coletadas, o alimento substituído e a mortalidade avaliada. As plantas foram trocadas a cada 10 dias por plantas cultivadas em casa-de-vegetação com cerca de 20 dias de idade. A partir dos dados coletados foi determinada a longevidade das fêmeas, o número de ovos e de ninfas produzidas por fêmea.

Biologia de B. tabidus com acesso à planta alimentado com ou sem presa. $\mathrm{O}$ desenvolvimento e a reprodução de $B$. tabidus foram estudados criando os predadores com presa e acesso as plantas de algodoeiro, caruru e feijão. As gaiolas para o confinamento dos insetos com presa e planta foram semelhantes àquelas utilizadas no experimento anterior. Os insetos e plantas foram mantidos em câmara climáticas do tipo B.O.D. à temperatura de $25 \pm 1^{\circ} \mathrm{C}, 68 \pm 5 \%$ de U.R. e fotofase de $12 \mathrm{~h}$. As plantas foram cultivadas de acordo com a metodologia citada anteriormente. Seis ninfas de segundo estádio $(<12 \mathrm{~h})$ foram confinadas com presas por gaiola com uma planta cada. As plantas utilizadas encontravam-se com aproximadamente 20 dias de idade e com folhas completamente desenvolvidas. Foram estudadas 60 ninfas por tratamento distribuídas em 10 repetições para cada tratamento (três tratamentos com presa mais planta de algodoeiro, feijão e caruru e, três tratamentos com as respectivas plantas sem presas). Para isolar o efeito mediado pela planta através da presa, foram empregadas pupas da presa alternativa $T$. molitor, uma vez que esta espécie não se alimenta da planta e é considerada adequada para o predador (Zanuncio et al. 1996). As plantas eram substituídas a cada 10 dias por plantas cultivadas em casa-de-vegetação como descrito anteriormente.

Foram realizadas avaliações para a determinação da viabilidade ninfal (porcentagem de ninfas que atingiram a fase adulta), duração da fase ninfal (o tempo decorrido entre a passagem para o segundo instar e a morte das mesmas ou muda para a fase adulta) e peso de adultos, daquelas confinadas sobre plantas com ou sem presa.

Para avaliar o efeito da planta na longevidade e reprodução de $B$. tabidus, os insetos recém-emergidos dos tratamentos de presa mais planta foram mantidos sobre as mesmas plantas que foram criadas e compuseram os níveis dos tratamentos presa mais planta e planta sem presa. A cada $48 \mathrm{~h}$, foi anotada a mortalidade, número de ovos e, posteriormente, o número de ninfas.

Análises. A frequência de predadores apresentando escolha entre plantas (algodoeiro, feijão e caruru), tanto machos e fêmeas, para alimentação foram analisados através do Proc Freq do SAS e interpretados pelo teste do quiquadrado. O tempo de alimentação, ganho de peso adquirido com a alimentação nas plantas foram submetidos à análise de variância (ANOVA) sendo os dados do tempo de alimentação e a longevidade transformados em $\log (\mathrm{x}+1)$ para atender os pré-requisitos de normalidade (Kolmogorov D: normal test) e homogeneidade de variância (Bartlett's test) e considerando plantas hospedeiras como fatores principais na ANOVA. A relação entre o ganho de peso e o tempo de alimentação foi obtida por análise de regressão. A longevidade de adultos e o número de ovos e de ninfas por fêmea oriundos do teste de escolha foram transformados quando necessários para atender os pré-requisitos da ANOVA e considerando plantas hospedeiras e disponibilidade de presa (com e sem presa) como fatores principais na ANOVA. Os resultados para o desenvolvimento ninfal, longevidade e fecundidade foram submetidos à análise de variância para a comparação do efeito das plantas hospedeiras e as médias comparadas pelo teste de Tukey HSD. Os resultados de longevidade foram transformados em $\log (\mathrm{x}+1)$ e o de número de ovos por fêmea corrigidos por raiz $(\mathrm{x}+0,5)$ para atender os pré-requisitos da ANOVA. Resultados não transformados são apresentados nas tabelas e gráficos. A sobrevivência de ninfas sem presas e confinadas nas plantas foi comparada empregando o método Kaplan-Meyer do Proc LIFETEST. Todas as análises foram conduzidas utilizando o pacote estatístico SAS (SAS Institute 
1999-2001) e ANOVA empregando o Proc GLM devido a variações no número de repetições.

\section{RESULTADOS}

Comportamento de escolha da planta por B. tabidus e efeito na reprodução. Entre os indivíduos, 57,8\% das fêmeas e 66,2\% dos machos testados responderam positivamente alimentandose das plantas no período de observação das 07 às 19h, após estarem $24 \mathrm{~h}$ sem alimento. A escolha entre as plantas de algodoeiro, caruru e feijão para alimentação por $B$. tabidus foi semelhante para os machos $\left(\div^{2}=1,42 ; \mathrm{P}=0,4918\right)$ e fêmeas $\left(\div^{2}=\right.$ $4,50 ; \mathrm{P}=0,1053)$. A proporção de machos que escolheram alimentar-se de plantas de algodoeiro, caruru e feijão foi de 36,$9 ; 38,1$ e $25 \%$ e; para fêmeas de 19,7; 50,8 e 29,5\%, respectivamente, havendo apenas diferença para a planta de caruru ao nível de $10 \%$ de significância. Além da escolha, o tempo de alimentação de machos e fêmeas desse predador, também, foi semelhante quanto à planta hospedeira escolhida $\left(\mathrm{F}_{2,139}=0,54 ; \mathrm{P}=0,5855\right)$ (Fig. 1A). Resultados semelhantes foram registrados para o ganho de peso pela alimentação nas plantas $\left(\mathrm{F}_{2,139}=0,25 ; \mathrm{P}=0,7781\right)$ (Fig. 1B), mas o ganho de peso para fêmeas foi maior $(35,65 \pm 2,04 \mathrm{mg})$ que para machos $(24,62$ $\pm 1,46 \mathrm{mg})\left(\mathrm{F}_{1,139}=20,24 ; \mathrm{P}<0,0001\right)$ (Fig. 1B). Além disso, o ganho de peso tanto de machos quanto de fêmeas do predador apresentou relação significativa com o tempo de alimentação nas plantas (Fig. 2).

A manutenção dos adultos nas respectivas plantas escolhidas caracterizou maior longevidade das fêmeas quando mantidas no algodoeiro e caruru e menor em plantas de feijão. O número de ovos por fêmea também apresentou diferença significativa, sendo maior para fêmeas confinadas com presa mais plantas de algodoeiro ou de caruru que em feijão. No entanto, o número de ninfas por fêmea apresentou diferença em relação à espécie de planta escolhida apenas ao nível de $10 \%$ de significância (Tabela I).

Como esperado, a disponibilidade de presa afetou significativamente os três parâmetros avaliados. Todas as características avaliadas como a longevidade $\left(\mathrm{F}_{1,56}=15,63 ; \mathrm{P}\right.$ $=0,0002)$, produção de ovos $\left(\mathrm{F}_{1,56}=174,36 ; \mathrm{P}<0,0001\right)$ e de

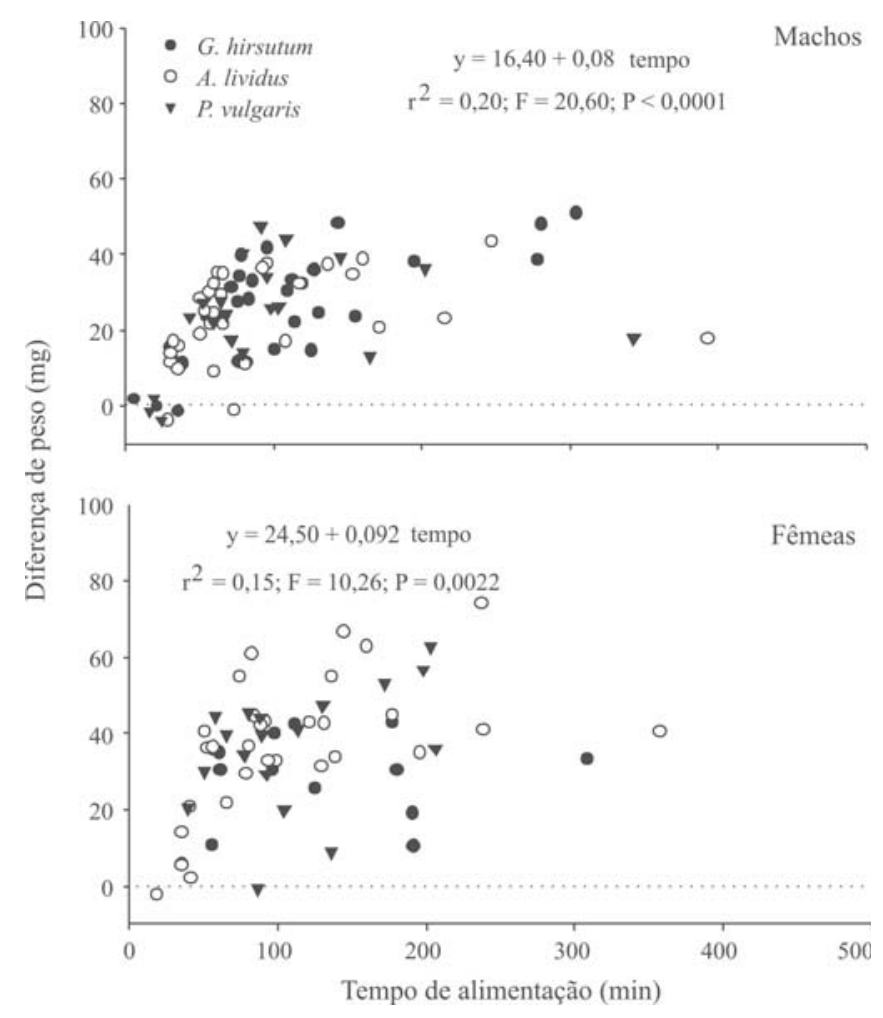

Fig. 2. Relação do ganho de peso (miligrama) de machos e fêmeas de Brontocoris tabidus com o tempo de alimentação (minutos) em três plantas hospedeiras de suas presas.

ninfas $\left(\mathrm{F}_{1,56}=134,48 ; \mathrm{P}<0,0001\right)$ foram superiores nos tratamentos com presa, independente da planta escolhida. Por outro lado, nenhum dos parâmetros avaliados foi afetado pela espécie de planta, estando os predadores sem acesso a presa, sendo que a longevidade, neste caso, não ultrapassou 22 dias (Tabela I).

Biologia de B. tabidus com acesso à planta alimentado com ou sem presa. A duração da fase ninfal de B. tabidus foi prolongada em, aproximadamente, um dia quando o inseto foi alimentado com presa mais planta de algodoeiro $\left(\mathrm{F}_{2,138}=4,06\right.$; $\mathrm{P}=0,0194$ ) (Tabela II). Por outro lado, o tipo de planta hospedeira não afetou a viabilidade ninfal $\left(\mathrm{F}_{2,27}=0,52 ; \mathrm{P}=\right.$

Tabela I. Número de ovos, ninfas e longevidade de fêmeas de Brontocoris tabidus mantidas em plantas com presa e sem presa, pupa de Tenebrio molitor, após teste de escolha para alimentação nas respectivas plantas. Temp.: $25 \pm 2^{\circ} \mathrm{C}$ e $12 \mathrm{~h}$ de fotofase.

\begin{tabular}{|c|c|c|c|}
\hline Dieta $^{1}$ & Número de ovos/우 & Número de ninfas/우 & Longevidade 우 (dias) \\
\hline \multicolumn{4}{|l|}{ Com presa } \\
\hline T. molitor + Gossypium hirsutum & $63,3 \pm 25,74 a b$ & $48,3 \pm 19,82 a$ & $26,3 \pm 3,57 \mathrm{a}$ \\
\hline T. molitor + Amaranthus lividus & $78,6 \pm 25,96 a$ & $51,2 \pm 16,88 a$ & $25,5 \pm 2,27 \mathrm{a}$ \\
\hline T. molitor + Phaseolus vulgaris & $31,4 \pm 14,00 \mathrm{~b}$ & $23,1 \pm 11,74 \mathrm{a}$ & $16,4 \pm 1,23 b$ \\
\hline $\begin{aligned} \text { Estatística, } & \mathrm{F}_{\mathrm{Gl}}{ }^{\mathrm{P}} \\
& \text { Sem presa }\end{aligned}$ & $\mathrm{F}_{2,56}=4,29=0,018$ & $\mathrm{~F}_{2,56}=3,02=0,056$ & $\mathrm{~F}_{2,56}=5,83=0,005$ \\
\hline Gossypium hirsutum & $3,8 \pm 1,91$ & $2,2 \pm 1,19$ & $21,0 \pm 2,31$ \\
\hline Amaranthus lividus & $2,6 \pm 1,50$ & $1,9 \pm 1,32$ & $21,3 \pm 2,60$ \\
\hline Phaseolus vulgaris & 0 & 0 & $14,2 \pm 1,39$ \\
\hline Estatística, $\mathrm{F}_{\mathrm{Gl}}^{\mathrm{P}}$ & $\mathrm{F}_{2,36}=2,90=0,06$ & $\mathrm{~F}_{2,36}=2,23=0,12$ & $\mathrm{~F}_{2,36}=2,66=0,08$ \\
\hline
\end{tabular}

${ }^{1}$ Médias ( \pm EP) seguidas da mesma letra, na coluna, não diferem entre si pelo teste de Tukey HSD $(P>0,05)$. 


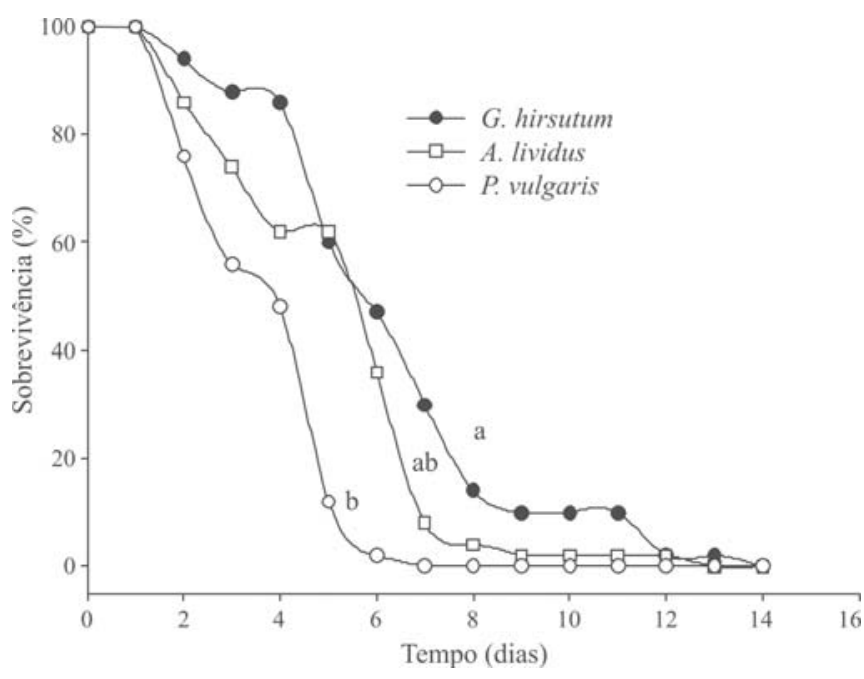

Fig. 3. Sobrevivência (\%) de ninfas de Brontocoris tabidus criadas sem presa e confinadas em Gossypium hirsutum, Amaranthus lividus e Phaseolus vulgaris. Temp.: $25 \pm 2{ }^{\circ} \mathrm{C}$ e $12 \mathrm{~h}$ de fotofase. Curvas de sobrevivência entre plantas diferem pelo Log-Rank Test $\left(\div^{2}=15,97\right.$; $=0,0003)$.

$0,6017)$ e o peso de adultos produzidos $\left(\mathrm{F}_{2,138}=0,14 ; \mathrm{P}=0,8696\right)$. A viabilidade ninfal variou de 73,3 a $88,3 \%$ e o peso de adultos de 118,4 a 160,5mg, para fêmeas, e de 114,3 a 123,2mg para machos, respectivamente (Tabela II). Entretanto, as fêmeas apresentaram maior peso $(157,5 \pm 2,62 \mathrm{mg})$ que os machos $(118,8$ $\pm 2,06 \mathrm{mg})\left(\mathrm{F}_{1,138}=134,43 ; \mathrm{P}<0,0001\right)$.

$\mathrm{Na}$ condição sem presa, o tempo de sobrevivência das ninfas foi de $6,5 \pm 0,37,5,4 \pm 0,32$ e 4,8 $\pm 0,22$ dias mantidas sobre as plantas de algodoeiro, caruru e feijão vagem, respectivamente. A sobrevivência de ninfas foi significativamente superior quando mantidas em algodoeiro em relação ao feijão (Fig. 3), porém sendo semelhante ao caruru e, entre caruru e feijão. O tempo máximo de sobrevivência sem presa obtido foi de 14 dias para uma ninfa confinada em algodoeiro.

A fecundidade de fêmeas criadas com presa mais plantas variou em função da planta hospedeira (Tabela II). Fêmeas de B. tabidus, quando alimentadas com presa mais algodoeiro ou caruru foram mais produtivas quando comparada àquelas mantidas em plantas de feijão $\left(\mathrm{F}_{2,30}=10,83 ; \mathrm{P}=0,0003\right) \mathrm{e}$, conseqüentemente, o mesmo resultado foi encontrado para $\mathrm{o}$ número de ninfas produzidas $\left(\mathrm{F}_{2,30}=9,05 ; \mathrm{P}=0,0008\right.$ ) (Tabela II). Na condição sem presa não houve produção de descendentes.

Fêmeas alimentadas de presa mais planta apresentaram maior longevidade $\left(\mathrm{F}_{2,30}=10,87 ; \mathrm{P}=0,0003\right)$ quando mantidas em algodoeiro e caruru e menor longevidade no feijão (Tabela II). Na condição sem presa mais planta, não houve diferença para a longevidade quanto à planta hospedeira $\left(\mathrm{F}_{2,30}=2,39 ; \mathrm{P}\right.$ $=0,1078)$, variando de 20 a 31,5 dias. Com relação à presença e ausência de presa, houve apenas diferença ao nível de $10 \%$ de significância na longevidade $\left(\mathrm{F}_{1,63}=3,89 ; \mathrm{P}=0,0529\right)$, sendo maior, como esperado, na condiçãa de disponibilidade de presa mais planta.

\section{DISCUSSÃO}

Os resultados mostram que $B$. tabidus não seleciona uma planta entre aquelas testadas para exercer a fitofagia e que adquire quantidade significativa de material durante a alimentação em plantas. O ganho de peso durante a alimentação da planta foi de, aproximadamente, 30 e $28 \%$ do peso corporal das fêmeas e machos, respectivamente. Este ganho de peso mostrou relação significativa com o tempo de alimentação para ambos (Fig. 2). A não preferência entre as plantas corrobora com o desempenho reprodutivo na maioria das características investigadas (Tabela II), resultando em igualdade de resultado entre as plantas. Ausência de impacto das plantas na biologia de B. tabidus seria o esperado por este ser um predador zoofitófago e não estritamente um fitófago. Entretanto, também, seria possível ocorrer impacto negativo sobre o predador caso a planta hospedeira apresentasse defesa contra este tipo de fitofagia. Assim, uma espécie de planta com características que acarretasse impacto negativo sobre percevejos predadores como aquelas resistentes a pragas poderia melhor distinguir o impacto da planta hospedeira no comportamento de escolha para alimentação em B. tabidus. Por exemplo, Matos-Neto et al. (2002) ao investigar o efeito da presa alimentada mais plantas de soja da cultivar IAC 17 resistente a herbivoria, encontraram efeito negativo para o período de oviposição e a fecundidade de $P$. nigrispinus. Entretanto, o efeito intrínsico da planta não foi o objetivo principal de nossa pesquisa, mas sim o comportamento de alimentação do predador e que demonstra ausência de preferência e da relação com o seu desempenho biológico desde que houve diferença no desempenho mas não na escolha da planta para alimentação.

Tabela II. Características da fase ninfal de Brontocoris tabidus criado com a presa Tenebrio molitor tendo acesso à plantas de algodoeiro, feijão e caruru e da fase adulta com presa, pupa de Tenebrio molitor. Temp.: $25 \pm 2{ }^{\circ} \mathrm{C}$ e $12 \mathrm{~h}$ de fotofase.

\begin{tabular}{|c|c|c|c|c|}
\hline \multirow[b]{2}{*}{ Dieta $^{1}$} & \multicolumn{4}{|c|}{ Fase ninfal } \\
\hline & \multicolumn{2}{|c|}{ Duração (dias) } & \multicolumn{2}{|c|}{ Viabilidade (\%) } \\
\hline \multicolumn{5}{|l|}{ T. molitor +} \\
\hline $\begin{array}{l}\text { Gossypium hirsutum } \\
\text { T. molitor }+\end{array}$ & \multicolumn{2}{|c|}{$25,0 \pm 0,28 \mathrm{a}$} & \multicolumn{2}{|c|}{$78,3 \pm 7,47 \mathrm{a}$} \\
\hline Amaranthus lividus & \multicolumn{2}{|c|}{$24,4 \pm 0,23 \mathrm{ab}$} & \multirow{2}{*}{\multicolumn{2}{|c|}{$88,3 \pm 4,34 \mathrm{a}$}} \\
\hline T. molitor + & & & & \\
\hline \multirow[t]{3}{*}{ Phaseolus vulgaris } & $24,1 \pm 0,2$ & $1 \mathrm{~b}$ & \multicolumn{2}{|c|}{$73,3 \pm 10,30 \mathrm{a}$} \\
\hline & \multicolumn{4}{|c|}{ Fase adulta } \\
\hline & $\begin{array}{l}\text { No. de } \\
\text { ovos/9+9 }\end{array}$ & & / $/ 9$ & $\begin{array}{c}\text { Longevidade } \\
\quad+\text { (dias) }\end{array}$ \\
\hline \multicolumn{5}{|l|}{ T. molitor +} \\
\hline Gossypium hirsutum & $282,7 \pm 79,87 \mathrm{a}$ & 156, & $2,07 \mathrm{a}$ & $44,7 \pm 5,98 \mathrm{a}$ \\
\hline $\begin{array}{l}\text { Amaranthus lividus } \\
\text { T. molitor }+\end{array}$ & $342,8 \pm 78,02 \mathrm{a}$ & 196 & $3,14 \mathrm{a}$ & $49,2 \pm 5,54 \mathrm{a}$ \\
\hline Phaseolus vulgaris & $30,3 \pm 14,42 b$ & 19,8 & $84 \mathrm{~b}$ & $17,7 \pm 2,21 \mathrm{~b}$ \\
\hline
\end{tabular}

${ }^{1}$ Médias $( \pm E P)$ seguidas da mesma letra, na coluna, não diferem entre si pelo teste de Tukey HSD $(P>0,05)$. 
A escolha pela planta hospedeira por B. tabidus pode ter ocorrido ao acaso e estar relacionada à presença da presa na planta e sua necessidade de umidade e alimento e não na qualidade da planta em si. O tempo de alimentação e o ganho de peso de B. tabidus em todas as plantas indicam que esse inseto adquiriu quantidades de água (seiva) e nutrientes semelhantes das três plantas. Diferenças no desempenho do predador podem estar relacionados a qualidade e proporção de cada material obtido das plantas (constituintes da seiva e do espaços intracelulares) e que merece futuros estudos para averiguação de quais materiais são ingeridos.

A menor duração no desenvolvimento ninfal de B. tabidus tendo acesso à plantas de feijão pode indicar que, apesar de o inseto apresentar um desenvolvimento mais rápido, isto resulta em efeito negativo posterior na sua biologia com uma menor fecundidade comparada ao algodoeiro e ao caruru. Desenvolvimento antecipado com posterior efeito nas características da fase adulta reflete deficiência na qualidade do alimento ingerido durante a fase imatura (Scriber \& Slanky Jr 1981). O efeito negativo de determinadas plantas para o desenvolvimento do predador advém da defesa intrínseca da planta, mediante compostos secundários (Cohen 1996). Por serem generalistas e utilizarem diversas presas e plantas, percevejos predadores podem sofrer com os aleloquímicos específicos de defesa das plantas contra herbívoros (Stamp et al. 1997). De acordo com Stamp \& Yang (1996), herbívoros generalistas são afetados por aleloquímicos específicos de plantas enquanto os herbívoros específicos co-evoluiram e não são afetados por estas defesas químicas. Desta forma, algum composto secundário expresso na variedade de feijão estudada e ausente nas outras plantas utilizadas deve ter acarretado efeito antibiótico sobre o predador e necessita ser estudado mais detalhadamente. Além disso, plantas de feijão possuem em sua constituição química inibidores de protease, que atuam na inibição de proteases como a tripsina, a quimiotripsina e outras durante a alimentação do inseto, fazendo com que este não consiga metabolizar as proteínas (Franco et al. 1999). Este resultado é interessante por se tratar de uma espécie de Fabaceae (antiga Leguminosae), que é a família de plantas preferida por pentatomídeos fitófagos e, também, suas vagens contribuírem no desenvolvimento e reprodução de percevejos predadores (Naranjo \& Stimac 1985; Coll 1996).

Sabe-se que a escassez de presa é fator limitante para o crescimento populacional de percevejos predadores, pois estes necessitam da presa para completar seu ciclo de vida. Por isto, B. tabidus não atingiu a fase adulta ou suas fêmeas produziram poucos ovos sem presa. Vale salientar que no teste de escolha (primeiro experimento), os adultos se alimentaram de presas durante dois dias antes de serem submetidos ao teste de escolha e, posteriormente, mantidos em condição de sem presa, o que garantiu a aquisição de reservas nutricionais para uma baixa produção de ovos (Tabela I). No entanto, este predador manteve-se vivo na fase adulta, em média, por duas a três semanas. De Clercq \& Degheele (1992) mencionaram que, na situação de escassez de presas, percevejos predadores mantêm a sobrevivência, reduzindo a capacidade reprodutiva e acumulando energia na espera de melhores condições no ambiente para a reprodução.

A fitofagia por predadores está, estreitamente, relacionada à alimentação em pólen e sementes/vagens partes consideradas como sendo rica em nitrogênio, comparado com o alimento obtido do xilema e floema (Jervis \& Kidd 1996; Eubanks et al. 2003). Em insetos predadores, a qualidade da planta como recurso alimentar, definida pela quantidade de nitrogênio, no entanto, não foi correlacionada com a magnitude dos efeitos positivos na longevidade e fecundidade de fêmeas (Eubanks \& Styrsky 2005). No entanto, entre partes da planta, sementes/vagens e pólen aumentaram a longevidade de insetos adultos mais que as folhas corroborando com a hipótese de maior disponibilidade de nitrogênio nestas partes resultando em melhoria no desempenho dos insetos. Estes resultados não são conclusivos considerando que fêmeas requerem fonte de nitrogênio para a produção de ovos e folhas tipicamente apresentam menos nitrogênio que sementes/ vagem ou pólen (Eubanks et al. 2003). Como neste estudo foram utilizadas plantas jovens e como B. tabidus provavelmente concentrou sua alimentação nas folhas das plantas, podemos sugerir que as diferenças na história de vida deste predador zoofitófago, entre as plantas estudadas, podem ser um resultado de diferenças na qualidade das folhas (i.e., seiva e demais componentes). Isto concorda com os resultados de Evangelista Júnior et al. (2004) que estudando seis espécies de plantas invasoras e o algodoeiro, também sem partes reprodutivas, encontraram diferenças no desempenho do percevejo predador $P$. nigrispinus.

Com base nos resultados, pode-se concluir que o predador B. tabidus não apresenta escolha para alimentação entre as plantas estudadas, o que favorece a sua ocorrência nos diferentes ecossistemas utilizando plantas como fonte de água e, possivelmente, de nutrientes. Entretanto, cada espécie de planta compondo a paisagem do agroecossistema, seja cultivada ou não, possui contribuição diferenciada na história de vida de percevejos predadores, como encontrado neste estudo para B. tabidus. Este resultado corrobora com a teoria de que a diversidade de plantas daninhas e cultivadas é importante para o estabelecimento de predadores nos agroecossistemas (Altieri 1989). Em particular, no caso dos percevejos predadores zoofitófagos como B. tabidus que podem se manter por duas a três semanas na ausência de presas.

Agradecimentos. À Coordenação de Aperfeiçoamento de Pessoal de Nível Superior (CAPES) pela concessão de bolsa (RRC) e ao Conselho Nacional de Pesquisa (CNPq) pela bolsa de produtividade (JBT).

\section{REFERÊNCIAS}

Altieri, M. A. 1989. Agroecologia: as bases científicas da agricultura alternativa. Rio de Janeiro, FASE/PTA, 237 p.

Armer, C. A.; R. N. Wiedenmann \& D. R. Bush. 1998. Plant feeding site selection on soybean by the facultatively phytophagous predator 
Orius insidiosus. Entomologia Experimentalis et Applicata 86 109-118.

Assis-Júnior, S. L.; T. V. Zanuncio; G. P. Santos \& J. C. Zanuncio. 1998. Efeito da suplementação de folhas de Eucalyptus urophylla no desenvolvimento e reprodução do predador Supputius cincticeps (Stal) (Heteroptera: Pentatomidae). Anais da Sociedade Entomológica do Brasil 27: 245-253.

Barcelos, J. A. V.; J. C. Zanuncio; A. C. Oliveira \& E. C. Nascimento. 1994. Performance em duas dietas e descrição dos adultos de Brontocoris tabidus (Signoret) (Heteroptera: Pentatomidae). Anais da Sociedade Entomológica do Brasil 23: 519-524.

Cohen, A. C. 1996. Plant feeding by predatory Heteroptera evolutionary and adaptational aspects of trophic switching, p. 1-17. In: O. Alomar \& R. N. Wiedenmann (eds.). Zoophytophagous Heteroptera: implications for life history and integrated pest management. Lanham, Entomological Society of America, $202 \mathrm{p}$.

Coll, M. 1996. Feeding and ovipositing on plants by an omnivorous insect predator. Oecologia 105: 214-220.

Coll, M. \& M. Guershon. 2002. Omnivory in terrestrial arthropods: mixing plant and prey diets. Annual Review of Entomology 47: 267-297.

De Clercq, P. \& D. Degheele. 1992. Influence of feeding interval on reproduction and longevity of Podisus sagitta ( Het.: Pentatomidae). Biocontrol 37: 583-590.

Eubanks, M. D. \& J. D. Styrsky. 2005. Effects of plant feeding on the performance of omnivorous predators. p. 148-177. In: M. A. Jervis \& N. A. C. Kidd (eds.). Insect natural enemies: practical approach to their study and evolution. London, Chapman \& Hall, $732 \mathrm{p}$.

Eubanks, M. D.; J. D. Styrsky \& R. F. Denno. 2003. The evolution of omnivory in heteropteran insects. Ecology 84: 2549-2556.

Evangelista Júnior, W. S.; M. G. C. Gondim Júnior; J. B. Torres \& E. J. Marques. 2004. Fitofagia de Podisus nigrispinus em algodoeiro e plantas daninhas. Pesquisa Agropecuária Brasileira 39: 413420 .

Franco, O. L.; F. R. Melo; M. C. M. Silva \& M. F. Grossi de Sá. 1999. Resistência de plantas a insetos. Biotecnologia Ciência e Desenvolvimento 10: $36-40$.

Jervis, M. A. \& N. A. C. Kidd. 1996. Phytophagy. p. 375-394. In: M. A. Jervis, N. A. C. Kidd (eds.). Insect natural enemies: practical approach to their study and evolution. London, Chapman \& Hall, $732 \mathrm{p}$.

Legaspi, J. C. \& B. C. Legaspi Jr. 2004. Does a polyphagous predator prefer prey species that confer reproductive advantage? Case study of Podisus maculiventris. Environmental Entomology 33: 14011409 .

Matos-Neto, F. C.; J. C. Zanuncio; M. C. Picanço \& I. Cruz. 2002. Reproductive characteristics of the predator Podisus nigrispinus fed with an insect resistant soybean variety. Pesquisa Agropecuária Brasileira 37: 917-924.

Molina-Rugama, A. J.; J. C. Zanuncio; J. B. Torres \& T. V. Zanuncio. 1997. Longevidad y fecundidad de Podisus nigrispinus (Heteroptera: Pentatomidae) alimentado con Musca domestica (Diptera: Muscidae) y fijol. Revista de Biologia Tropical 45: $1125-1130$

Naranjo, S. E. \& J. L. Stimac. 1985. Development, survival, and reproduction of Geocoris punctipes (Hemiptera: Lygaeidae): effects of plant feeding on soybean and associated weeds. Environmental Entomology 14: 523-530.
Naranjo, S. E. \& R. L. Gibson. 1996. Phytophagy in predaceus Heteroptera: effects on life history and population dynamics, p. 57-93. In: O. Alomar \& R. N. Wiedenmann (eds.). Zoophytophagous Hetroptera: implications for life history and integrated pest management. Lanham, Entomological Society of America, $202 \mathrm{p}$.

Oliveira, I.; J. C. Zanuncio; J. E. Serrão; T. V. Zanuncio; T. B. M. Pinon \& M. C. Q. Fialho. 2005. Effect of female weight on reproductive potential of the predator Brontocoris tabidus (Signoret, 1852) (Heteroptera: Pentatomidae). Brazilian Archives of Biology and Technology 48: 25-301.

Oliveira, J. E. M.; J. B. Torres; A. F. Carrano-Moreira \& R. Barros. 2002. Efeito de plantas do algodoeiro e do tomateiro, como complemento alimentar, no desenvolvimento e na reprodução do predador Podisus nigrispinus (Dallas) (Heteroptera: Pentatomidae). Neotropical Entomology 31: 101-108.

Ruberson, J. R.; M. J. Tauber \& C. A. Tauber. 1986. Plant feeding by Podisus maculiventris (Heteroptera: Pentatomidae): effect on survival, development, and preoviposition period. Environmental Entomology 15: 894-897.

SAS Institute. 1991-2000. User's guide, version 8.02, TS level 2MO. Cary, SAS Institute Inc., NC.

Scriber, J. M. \& F. Slanky Jr. 1981. The nutritional ecology of immature insects. Annual Review of Entomology 26: 183-211.

Stamp, N. E. \& Y. Yang. 1996. Response of insect herbivores to multiple allelochemical under different thermal regimes. Ecology 77: 10881102 .

Stamp, N. E.; Y. Yang \& T. L. Osier. 1997. Response of an insect predator to prey fed multiple allelochemicals under representative thermal regimes. Ecology 78: 203-214.

Stoner, A.; A. M. Metcalfe \& R. E. Weeks. 1974. Plant feeding by a predaceous insect, Podisus acutissimus. Environmental Entomology 3: 187-188.

Tillman, P. G. \& B. G. Mullinix Júnior. 2003. Effect of prey species on plant feeding behavior by the big-eyed bug, Geocoris punctipes (Say) (Heteroptera: Geocoridae), on cotton. Environmental Entomology 32: 1399-1403.

Torres, J. B.; J. C. Zanuncio \& M. A. Moura. 2006. The predatory stinkbug Podisus nigrispinus: biology, ecology and augmentative releases for lepidopteran larval control in Eucalyptus in Brazil. Biocontrol News and Information 27: 1-15.

Zanuncio, J. C.; J. L. D. Saavedra; H. N. Oliveira; D. Degheele \& P. De Clercq. 1996. Development of the predatory stinkbug Brontocoris tabidus Signoret (Hemiptera: Pentatomidae) on defferent proportions of an artificial diet and pupae of Tenebrio molitor L. (Coleoptera: Tenebrionidae). Biocontrol Science and Technology 6: 619-626.

Zanuncio, J. C.; T. V. Zanuncio; R. N. C. Guedes \& F. S. Ramalho. 2000. Effect of feeding on three Eucalyptus species on the development of Brontocoris tabidus (Het.: Pentatomidae) fed with Tenebrio molitor (Col.: Tenebrionidae). Biocontrol Science and Technology 10: 443-450.

Zanuncio, J. C.; W. P. Lemos; M. C. Lacerda; T. V. Zanuncio; J. E. Serrão \& E. Bauce. 2006. Age-dependent fecundity and fertility life tables of the predator Brontocoris tabidus (Heteroptera: Pentatomidae) under field conditions. Journal of Economic Entomology 99: 401-407. 